\title{
5-Azacytidine treatment results in nuclear exclusion of DNA methyltransferase-1, as well as reduced proliferation and invasion in human cytomegalovirus-infected glioblastoma cells
}

\author{
ATOSA ESTEKIZADEH ${ }^{1,2}$, NATALIA LANDÁZURI ${ }^{2}$, MATTIA RUSSEL PANTALONE $^{2}$, BELGHIS DAVOUDI $^{2}$, \\ LI-FU HU ${ }^{3,4}$, IMRAN NAWAZ ${ }^{3-5}$, GIUSEPPE STRAGLIOTTO ${ }^{2,6}$, \\ TOMAS J. EKSTRÖM ${ }^{1}$ and AFSAR RAHBAR ${ }^{2}$ \\ ${ }^{1}$ Department of Clinical Neuroscience, Center for Molecular Medicine; ${ }^{2}$ Department of Medicine (Solna), \\ Division of Microbial Pathogenesis, BioClinicum; ${ }^{3}$ Department of Microbiology, Tumor and Cell Biology, \\ Karolinska Institutet, 17165 Stockholm, Sweden; ${ }^{4}$ Shenzhen People's Hospital, Shenzhen, Guangdong 518020, \\ P.R. China; ${ }^{5}$ Department of Microbiology, University of Balochistan, Quetta 87300, Pakistan; \\ ${ }^{6}$ Division of Neurosurgery, Karolinska University Hospital, 17176 Stockholm, Sweden
}

Received May 16, 2018; Accepted February 7, 2019

DOI: 10.3892/or.2019.7074

\begin{abstract}
Glioblastoma (GBM) is the most aggressive form of brain tumor in adults, with a devastating outcome. Emerging evidence shows that human cytomegalovirus (HCMV) proteins and nucleic acids are present in GBM tissues. DNA methylation is important for the initiation and progression of cancer and is an established host response against invading nucleic acids. The expression and localization of DNA methyltransferase 1 (DNMT-1) was assessed, and the effects of DNA methylation inhibitor 5-azacytidine (5AZA) were analyzed in the context of the viral replication, proliferation and invasion capacities of HCMV-infected GBM U343MG cells. In addition, the expression of various HCMV proteins and DNMT-1 was examined in GBM tissue specimens obtained from five patients. DNMT-1 was localized in the nucleus of cells expressing $\mathrm{HCMV}$-immediate early, whereas in cells expressing HCMV-glycoprotein $\mathrm{gB}(\mathrm{gB})$, extranuclear/cytoplasmic localization was observed. This was also observed in vitro in U343MG cells. In addition, DNMT-1 was localized to the extranuclear/cytoplasmic space of cells lining blood vessel walls within the GBM tumors. Treatment of infected U343MG cells with 5AZA did not affect viral replication, but reduced cell invasion and proliferation $(\mathrm{P}=0.05$ and $\mathrm{P}<0.0001$,
\end{abstract}

Correspondence to: Dr Afsar Rahbar, Department of Medicine (Solna), Akademiska Stråket 1, BioClinicum, Karolinska Institutet, 17176 Stockholm, Sweden

E-mail: afsar.rahbar@ki.se

Key words: human cytomegalovirus, DNA methyltransferase-1, 5-azacytidine, proliferation, invasion respectively). However, 5AZA treatment of uninfected cells did not affect cell invasion $(\mathrm{P}=0.09)$, but proliferation was significantly reduced $(\mathrm{P}<0.0001)$. These findings may be of importance in further investigations aimed at using DNA methylation and viral inhibitors in GBM therapy.

\section{Introduction}

Human cytomegalovirus (HCMV) is a member of the Herpesviridae family with a genome size of $236 \mathrm{~kb}$, the largest of any known human virus $(1,2)$. The HCMV genome encodes 750 proteins, of which $\sim 50$ are involved in the construction of new viral particles. The vast majority of viral proteins function in regulating important host functions that assist the virus to co-exist with its host (3). During evolution, HCMV has developed unique mechanisms to adapt to the human immune system, allowing it to maintain a latent phase in $\mathrm{CD} 34^{+}$myeloid progenitor cells (4). Originally it was thought that HCMV is completely inactive during latency. However, various viral transcripts and proteins appear to be induced during this phase, including latency unique natural antigen (LUNA) (5), G-protein coupled receptor homolog US28 (US28), viral interleukin (IL)-10 homolog (UL111A) and membrane glycoprotein UL144 (UL144) (6). These viral proteins affect the immune system. For example, LUNA stimulates the immune response (5), US28 is associated with inflammation, and UL111 A and UL144 are involved in HCMV immune evasion (7). Furthermore, different biological conditions were shown to be central in the reactivation of latent HCMV, including inflammation, immune suppression, other infections and epigenetic modifications $(8,9)$. The critical factor in transcriptional reactivation of latent HCMV is terminal differentiation of latently infected monocytes into macrophages or dendritic cells $(8,10)$. Accumulating evidence suggests an association between histone modification, chromatin modulation, HCMV genome 
transcription and replication, both during latency and lytic infection $(9,11,12)$. Additionally, previous studies have shown that histone deacetylases (HDACs) are bound to the HCMV promoter and HDAC activity results in repression of viral gene expression and latency (13-15). However, the involvement of host cell epigenetic alterations in HCMV reactivation remains poorly understood.

Previous studies have revealed the frequent presence of HCMV nucleic acid sequences and proteins in malignant brain tumors, including glioblastoma multiforme (GBM), medulloblastoma (MB) and neuroblastoma (16-18). GBM is the most aggressive brain tumor in adults, with fatal outcomes. Despite aggressive surgery and advanced therapy, the median overall survival of these patients is $\sim 14$ months (19). The risk factors for GBM are unknown but environmental exposures to radiation, vinyl chloride and pesticides have been considered in the initiation of GBM $(20,21)$. Traumatic brain injury and consequent inflammation have also been suggested in the etiology of GBM, although this is still controversial (22).

HCMV has not been classified as an oncogenic virus as it does not have the ability to transform cells, but the term oncomodulation has been used to describe the actions of HCMV in tumors (23). HCMV may modulate cancer progression through interactions with potential oncogenic factors within the host cells, such as p53, retinoblastoma protein $(\mathrm{Rb})$ and cyclins, resulting in uncontrolled cell proliferation (24). Our recent report found that HCMV replication significantly increased in HCMV infected MB cells treated with 5-azacytidine (5AZA) (25). Since epigenetic drugs are suggested as agents of cancer therapy (26) and GBM tissues have been shown to be frequently positive for HCMV proteins and nucleic acids, the present study investigated the viral replication, proliferation and invasion capacity of 5AZA-treated HCMV-infected GBM cells (U343MG), and examined the expression of DNMT-1 and HCMV proteins in GBM tissue specimens.

\section{Materials and methods}

Cell culture and HCMV infection. U343MG and MRC-5 cells were obtained from American Type Culture Collection (Manassas, VA, USA). All cells (1.5-3.0x105) were cultured under the following conditions, unless otherwise stated: Dulbecco's modified Eagle's medium containing $10 \%$ heat-inactivated fetal bovine serum (FBS), $100 \mathrm{U} / \mathrm{ml}$ penicillin and $100 \mu \mathrm{g} / \mathrm{ml}$ streptomycin (all from Gibco; Thermo Fisher Scientific, Inc., Waltham, MA, USA) at $37^{\circ} \mathrm{C}$ in a humidified $5 \% \mathrm{CO}_{2}$. Cells were infected with $\mathrm{HCMV}$ clinical isolate (strain VR1814; a kind gift from Dr Giuseppe Gerna, University of Pavia, Pavia, Italy) at a multiplicity of infection (MOI) of 5 for U343MG cells and a MOI of 1 for MRC-5 cells, for 3 days. In all experiments, cells cultured without HCMV were used as the negative control. Transcripts from HCMV infected MRC-5 cells were used as positive control [with an infection efficiency for HCMV-Major Immediate-Early gene equal to $\mathrm{C}_{\mathrm{t}}=21.2$ from quantitative TaqMan polymerase chain reaction (qPCR); protocol described below], since MRC-5 cells are permissive for infection using the HCMV strain VR1814 ( $>80 \%$ infected cells). The number of cells used in different treatment experiments was $1.5-3.0 \times 10^{5}$. All experiments were performed with three independent repeats.
Immunofluorescence staining. Uninfected and HCMV infected U343MG cells $\left(1.5-3.0 \times 10^{5}\right)$ were fixed in ice-cold acetone:methanol (1:1) for $10 \mathrm{~min}$ (Sigma-Aldrich; Merck KGaA, Darmstadt, Germany). Immunofluorescence staining of different proteins was performed as described previously, with minor modifications (25). Endogenous non-specific binding was blocked using Protein Block, Serum-Free (cat. no. X0909; Dako; Agilent Technologies, Inc., Santa Clara, CA, USA). The cells were incubated with the following primary antibodies: Mouse monoclonal antibody to immediate early (IE-1 and IE-2; 1:100; cat. no. 11-003; ARGENE; bioMérieux, Verniolle, France); mouse monoclonal antibody to glycoprotein $\mathrm{gB}$ (gB; 1:50; cat. no. P1216; EastCoast Bio, Inc., North Berwick, ME, USA); rabbit polyclonal antibody to DNMT-1 (1:500; cat. no. ab19905; Abcam). Binding of primary antibodies to specific proteins was detected by incubation with Alexa Fluor 488-conjugated goat anti-mouse (1:500; cat no: A-11001; Molecular Probes; Thermo Fisher Scientific, Inc.) and Texas red-conjugated goat anti-rabbit (1:500, cat no: T-6391, Molecular Probes; Thermo Fisher Scientific, Inc.) as secondary antibodies for $45 \mathrm{~min}$ at room temperature. Incubation with PBS instead of primary antibodies served as negative control. Ready to use fluorescence mounting medium (with DAPI; Vectashield; Vector Laboratories, Inc., Burlingame, CA, USA) was used for $1 \mathrm{~min}$ for nuclear staining and mounting. Staining was evaluated by confocal microscopy (scale bar, $50 \mu \mathrm{m}$; Leica TCS SP5; Leica Microsystems GmbH, Wetzlar, Germany).

RNA extraction and reverse transcription-quantitative $P C R$ (RT-qPCR). A RNeasy kit (Qiagen AB, Sollentuna, Sweden) was used to extract the total RNA from cells according to the manufacturer's instructions. The RNA concentration was measured using a NanoDrop 1000 spectrophotometer (Thermo Fisher Scientific, Inc.). RNA (500 ng) was converted to cDNA using the SuperScript III First-Strand Synthesis system (Invitrogen; Thermo Fisher Scientific, Inc.) according to the manufacturer's instructions. TaqMan qPCR with specific target primers and probes (FAM fluorophore) was performed using a 7900HT Fast Real-Time PCR system (Applied Biosystems; Thermo Fisher Scientific, Inc.). Target primers and probes used in this study were as follows, as described previously (25): HCMV-IE (forward primer, 5'-TGACGAGGGCCC TTCCT-3' and reverse primer, 3'-CCTTGGTCACGGGTG TCT-5'; probes, FAM-AAGGTGCCACGGCCCG-NFQ) and HCMV-gB (forward primer, 5'-GCTACCGCCCTACCTCAA G-3' and reverse primer, 3'-CGCCAACGGCCTTTCC-5'; probes, FAM-CCCAGGCCGCTCATG-NFQ) and DNMT-1 (assay ID, Hs00945875_m1; Life Technologies; Thermo Fisher Scientific, Inc.). RNase P (housekeeping gene; assay ID, 4316844; Life Technologies; Thermo Fisher Scientific, Inc.) was used for normalization. All assays were performed according to the manufacturer's protocols.

The qPCR thermocycler was initiated with polymerase activation at $95^{\circ} \mathrm{C}$ for $20 \mathrm{sec}$ followed by 40 cycles of denaturation at $95^{\circ} \mathrm{C}$ for $1 \mathrm{sec}$ and annealing/extension at $60^{\circ} \mathrm{C}$ for $20 \mathrm{sec}$. PCR results were analyzed with SDS version 2.4 software (Thermo Fisher Scientific, Inc.). The $\Delta \mathrm{Ct}$ method was used for calculation of $\mathrm{Ct}$ values for different transcripts. The $2^{-\Delta \Delta \mathrm{Ct}}$ method was used to quantify relative fold changes (27). 
Table I. GBM patient characteristics.

\begin{tabular}{|c|c|c|c|c|c|c|c|}
\hline Patient & GBM & Sex & Age & P53 mutations & KI-67 (\%) & TTP (months) & OS (months) \\
\hline 1 & Primary & Female & 62 & Negative & 80 & NR & 3 \\
\hline 2 & Primary $^{\mathrm{a}}$ & Male & 53 & ND & 25 & 8 & 14.5 \\
\hline 3 & Primary & Male & 75 & Positive & 25 & 3 & 11 \\
\hline 4 & Primary & Male & 62 & Negative & 35 & 9 & 15.5 \\
\hline 5 & Secondary ${ }^{\mathrm{b}}$ & Female & 34 & Positive & 70 & NR & $38^{\mathrm{c}}, 8^{\mathrm{d}}$ \\
\hline
\end{tabular}

${ }^{\mathrm{a} O}$ Oligodendrogliomas; ${ }^{\mathrm{b}} \mathrm{GBM}$ progressed from astrocytoma grade II; ${ }^{\mathrm{c}}$ time until secondary GBM occurrence; ${ }^{\mathrm{d} O S}$ following secondary GBM. GBM, glioblastoma multiforme; ND, not determined; NR, not relevant; TTP, time to tumor progression.

5AZA treatment. U343MG cells were treated with 5AZA (5-Aza-2'-deoxycytidine; $10 \mu \mathrm{M}$; Sigma-Aldrich; Merck KGaA) for 3 days and subsequently infected with HCMV-VR1814 at a MOI of 5 for 3 days, whilst maintaining the 5AZA concentration. Cells were fixed in ice-cold acetone:methanol (1:1) for 10 min 3 days post-infection (dpi) and were kept at $-20^{\circ} \mathrm{C}$ until further experimentation.

Ganciclovir $^{\circledR}$ treatment. Ganciclovir $^{\circledR}$ (2 mM; cymevene; Apoteket AB, Stockholm, Sweden) was used to treat U343MG cells at the time of HCMV infection. Cells were fixed as described above.

Invasion assay. Cells were plated on 6-well plates (day 0), untreated $\left(2 \times 10^{5}\right)$ or treated with 5-AZA $\left(3 \times 10^{5}\right)$ from days $0-5$. On day 2, cells were infected (or not) with HCMV (MOI of 5 ). On day 5, cells were detached from the plates and used for the invasion assay. Invasion assays were performed using CytoSelect $^{\mathrm{TM}}$ 6-Well Cell Migration and Invasion assay kit (8 $\mu \mathrm{m}$; Colorimetric Format; Cell Biolabs, Inc., San Diego, CA, USA) according to the manufacturer's instructions. Briefly, under sterile conditions, untreated or 5AZA treated uninfected or HCMV infected cells in serum free culturing medium were plated on each insert and culturing media containing 10\% FBS was added in the lower well of the migration plate. The plates were incubated at $37^{\circ} \mathrm{C}\left(5 \% \mathrm{CO}_{2}\right)$ for $24 \mathrm{~h}$. Non-migrated cells on the upper side of the inserts were gently removed by wet cotton-tipped swabs. The inserts were transferred to a new wells and $200 \mu 1$ extraction solution (provided in the kit) was added followed by a 10 min incubation on an orbital shaker. Finally, $100 \mu 1$ from each sample was transferred to a 96-well microtiter plate. The optical density of the resulting extracts was measured at $\lambda=560 \mathrm{~nm}$ and normalization was performed with the proliferation values at time 0 before treatment.

Proliferation assay. Uninfected and HCMV infected U343MG cells, untreated or treated with 5AZA, were seeded in 96-well culture plates (1,000/well) and allowed to attach. At 3 days post-treatment, cell viability was assessed using the CellTiter $96{ }^{\circledR}$ AQueous Non-Radioactive Cell Proliferation assay (Promega Corporation, Madison, WI, USA) according to the manufacturer's instructions. The absorbance was recorded at $\lambda=490$ and normalization was performed with the proliferation values at day 2 , prior to treatment.
Immunohistochemical staining (IHC). Paraffin embedded human GBM tumor tissue sections from five patients (four patients with primary and one patient with secondary GBM; one primary GBM patient had chromosome $1 \mathrm{p} 19 \mathrm{q}$ deletion; Table I) were retrospectively and randomly obtained between October 2009 and November 2010 from the Department of Pathology at Karolinska University Hospital (Stockholm, Sweden). The inclusion criterion was GBM tumor tissue. No recruitment period or other control samples/groups were defined. GBM tumor tissue samples were obtained at surgery at Karolinska University Hospital from adult GBM patients with written informed consent. Samples were stored in a biorepository and anonymized. Ethical permission was obtained from the Ethics Committee at the Karolinska Institutet (Stockholm, Sweden; Dnr. 2008/628-31).

Immunohistochemical staining of the tissues was performed as described previously, with some minor modifications $(16,17)$. Following deparaffinization of the tissue specimens in xylene and rehydration in a series of decreasing ethanol concentrations, tissue sections were permeabilized with pepsin (Nordic BioSite AB, Täby, Sweden) and citrate buffer (Bio-Genex Laboratories, Fremont, CA, USA). Endogenous peroxidase activity was blocked with $3 \%$ $\mathrm{H}_{2} \mathrm{O}_{2}$ (Sigma-Aldrich; Merck KGaA) and the sections were treated with an avidin/biotin blocking kit (cat. no. X0590; Dako; Agilent Technologies, Inc.), Fc receptor blocker (cat. no. NB309; Innovex Biosciences, Richmond, CA, USA) and Background Buster (cat. no. NB306; Innovex Biosciences) to eliminate non-specific binding. All blocking steps were performed according to the kit protocols. ImmPRESS reagent kits (cat. no: MP-7401 and MP-7402; Vector Laboratories, Inc.) and diaminobenzidine were used to detect binding of primary antibodies to targeted proteins. The primary antibodies used in IHC were as follows: HCMV-IE (cat. no. MAB810R; Merck KGaA), HCMV-Late (cat. no. MAB8127; Merck KGaA), HCMV-gB (a kind gift from Dr William Britt, University of Alabama, USA), DNMT-1 (cat. no. ab19905, Abcam). For the negative control group, primary antibodies were omitted. For scanning of IHC sections, a Hamamatsu Nano Zoomer-XR Digital slide scanner C12000 (Hamamatsu Phototonics, Hamamatsu, Japan) was employed, with visualization using the Nano Zoomer Digital Pathology (NDP) viewer software (U12388-01; NDP.view2 Viewing; Hamamatsu Phototonics). 

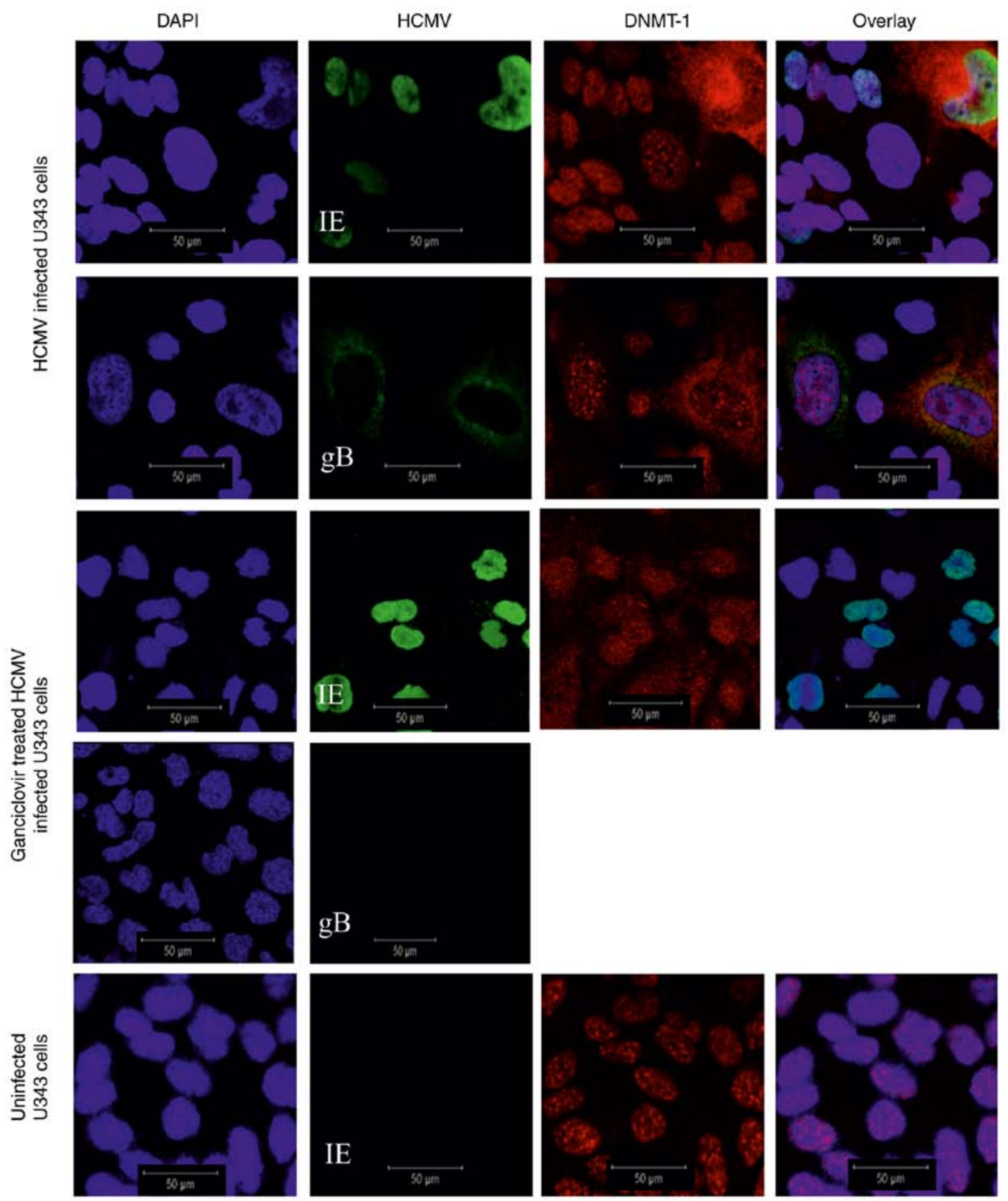

Figure 1. Cytoplasmic expression of DNMT-1 in HCMV-infected U343MG GBM cells expressing HCMV-gB protein. Expression of DNMT-1 in the nucleus of HCMV-IE positive cells and in the cytoplasm of HCMV-gB expressing U343MG GBM cells. DNMT-1 was expressed in the nucleus of Ganciclovir ${ }^{\circledR}$-treated HCMV-infected cells expressing HCMV-IE, but not HCMV-gB proteins (scale bar, $50 \mu \mathrm{m}$ ). DNMT-1, DNA methyltransferase 1; HCMV, human cytomegalovirus; GBM, glioblastoma; gB, glycoprotein $\mathrm{gB}$; IE, immediate early.

Statistical analysis. All analyses were performed using GraphPad Prism version 6 (GraphPad Software, Inc., La Jolla, CA, USA). $\mathrm{P}<0.05$ was considered to indicate a statistically significant difference. Unpaired Student's t-test or one-way analysis of variance followed by Dunnett's multiple comparisons test were used to assess the statistical significance between different variables. Data are presented as the mean \pm standard error of the mean. All experiments were performed with three independent repeats.

\section{Results}

Cytoplasmic expression of DNMT-1 in HCMV infected U343MG cells expressing viral late genes. U343MG GBM cells were infected with HCMV for 3 days in order to observe full length viral replication. Expression of DNMT-1 was detected in the nucleus of uninfected and HCMV-IE expressing cells (i.e. cells displaying immediate early phases of HCMV infection), but was evidently expressed exclusively in the extranuclear space of HCMV-gB positive cells (i.e. cells displaying later phases of HCMV infection) (Fig. 1). At $3 \mathrm{dpi}, 18$ and $4 \%$ of the U343MG cells expressed HCMV-IE and HCMV-gB proteins, respectively, $(\mathrm{P}=0.002)$ and the HCMV-IE transcript was present at higher levels than HCMV-gB transcripts in the infected cells $(\mathrm{P}=0.0007$; Fig. 2A and B). DNMT-1 transcript levels did not change significantly in HCMV-infected cells compared to uninfected cells (Fig. 2C). 


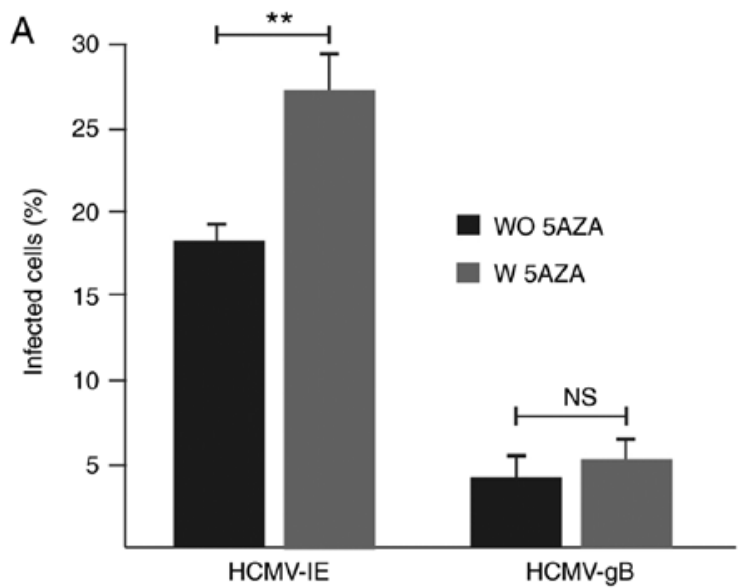

B
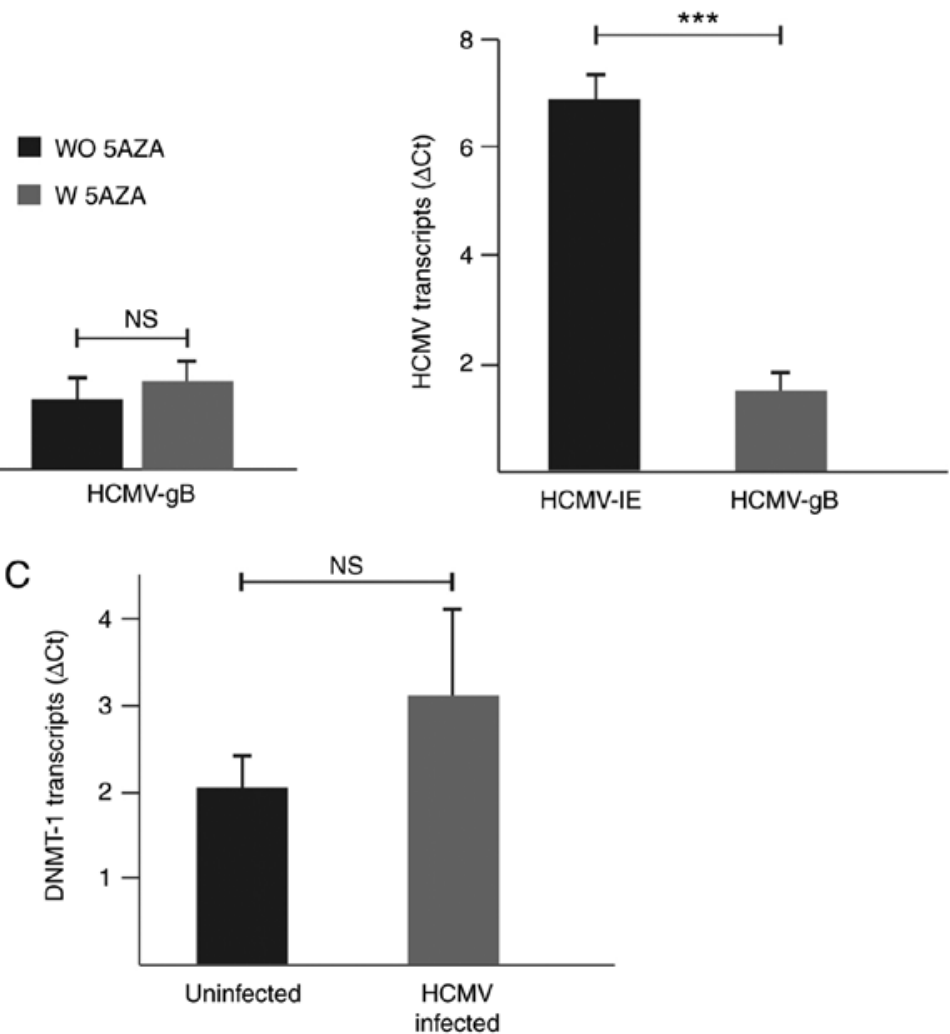

Figure 2. Expression of HCMV proteins and transcripts and in U343MG GBM cells. (A) Percentage of U343MG cells expressing HCMV-IE and HCMV-gB proteins in U343MG GBM cell group. (B) Detection of HCMV-IE and HCMV-gB transcripts at 3 days post-infection. (C) Unchanged levels of DNMT-1 transcripts in HCMV-infected cells compared with uninfected cells. ${ }^{* *} \mathrm{P}<0.01,{ }^{* * * *} \mathrm{P}<0.001$. DNMT-1, DNA methyltransferase 1; HCMV, human cytomegalovirus; GBM, glioblastoma; gB, glycoprotein gB; IE, immediate early; NS, not significant.

5AZA treatment does not modulate transcription levels of viral genes. U343MG GBM cells were treated with 5AZA and subsequently infected with HCMV for 3 days. Untreated and/or uninfected cells were used as the controls. In 5AZA-treated HCMV-infected cells, HCMV-IE and HCMV-gB proteins were detected in 27 and $5 \%$ of the cells, respectively ( $\mathrm{P}=0.002$; Fig. 2A). No significant differences were observed in the levels of HCMV-IE and HCMV-gB viral transcripts, as well as DNMT-1 transcripts in 5AZA-treated HCMV-infected cells compared with untreated HCMV-infected cells (data not shown; HCMV-IE, $\mathrm{P}=0.2$; HCMV-gB, P=0.4; DNMT-1, P=0.1).

Antiviral treatment prevents extranuclear localization of DNMT-1. Uninfected and HCMV-infected U343MG GBM cells were treated with the antiviral drug Ganciclovir ${ }^{\circledR}$ in order to inhibit the expression of late viral genes. As expected, $\mathrm{HCMV}-\mathrm{gB}$ protein expression was not detected in the Ganciclovir ${ }^{\circledR}$-treated cells (Fig. 1). DNMT-1 expression was retained in the nuclei of HCMV-infected antiviral treated cells (Fig. 1).

U343MG cell invasion is significantly decreased by HCMV infection. Untreated HCMV-infected U343MG cell invasion was significantly decreased compared with the untreated uninfected cells $(\mathrm{P}=0.03)$. Cell invasion was further decreased with 5AZA treatment in infected cells, compared with the untreated uninfected cells $(\mathrm{P}=0.0009)$. However, a borderline significant decrease in invasive ability was observed in treated infected cells compared with the untreated infected cells $(\mathrm{P}=0.05)$. A significant difference in invasion was observed between the 5AZA-treated uninfected and HCMV-infected cells $(\mathrm{P}=0.02$; Fig. 3A).

5AZA treatment significantly decreases the proliferation of uninfected and HCMV-infected U343MG GBM cells. The proliferation of 5AZA-treated uninfected and HCMV-infected cells was significantly decreased compared with the untreated uninfected cells $(\mathrm{P}<0.0001)$ and untreated HCMV infected cells $(\mathrm{P}<0.0001)$, respectively (Fig. 3B). No significant differences in proliferation were detected between the untreated uninfected cells, compared with the untreated infected cells $(\mathrm{P}=0.4)$. The proliferation ability of 5AZA-treated uninfected cells was significantly decreased compared with the 5AZA-treated HCMV-infected cells $(\mathrm{P}=0.02)$. Furthermore, the proliferation of 5AZA-treated HCMV-infected cells was significantly decreased compare to untreated uninfected cells $(\mathrm{P}=0.0001$; Fig. 3B).

Cytoplasmic expression of DNMT-1 in the cells of vessel walls within the GBM. Expression of HCMV-IE, HCMV-gB, HCMV-late and DNMT-1 proteins was examined in available GBM tissues sections obtained from 5 patients by IHC (Table II). While the expression of HCMV-gB and 
Table II. MGMT methylation status and detection of HCMV-proteins and DNMT-1 in GBM tissues by immunohistochemical staining.

\begin{tabular}{|c|c|c|c|c|c|c|}
\hline \multirow[b]{2}{*}{ Patient } & \multicolumn{3}{|c|}{ HCMV } & \multirow{2}{*}{$\frac{\text { MGMT }}{\text { Methylation }}$} & \multicolumn{2}{|c|}{ DNMT-1 } \\
\hline & IE & Late & $\mathrm{gB}$ & & Nucleus & Cytoplasmic \\
\hline 1 & $4+$ & $2+$ & $1+$ & ND & Tumor cells & Vessel cells \\
\hline 2 & $4+$ & $2+$ & $1+$ & Unmethylated & Tumor cells & Tumor and vessel walls \\
\hline 3 & $4+$ & $1+$ & $1+$ & Unmethylated & Tumor cells & Vessel walls \\
\hline 4 & $3+$ & $2+$ & $1+$ & Methylated & Tumor cells & Vessel walls \\
\hline 5 & $4+$ & $2+$ & $1+$ & Methylated & Tumor cells & Vessel walls \\
\hline
\end{tabular}

0 , negative, $1+, 5-10 \%$ positive cells; $2+, 10-25 \%$ positive cells; $3+, 25-50 \% ; 4+, 50-75 \%$ positive cells. HCMV, human cytomegalovirus; IE, immediate early; gB, glycoprotein-B; MGMT, methylated-DNA-protein-cysteine methyltransferase; ND, not determined.
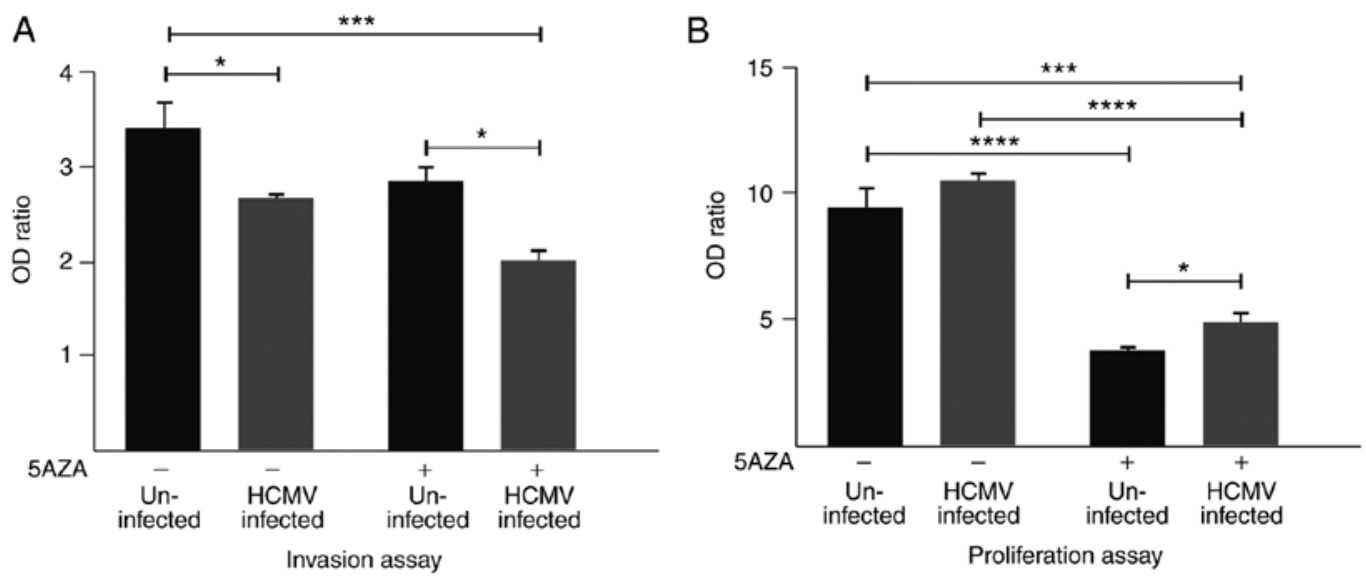

Figure 3. U343MG cell invasion is significantly decreased by HCMV infection. (A) Invasion assays were performed in U343MG GBM cells. Invasion was significantly decreased in 5AZA-treated HCMV-infected cells, compared with untreated or treated uninfected cells. (B) Proliferation assays were performed in U343MG GBM cells. Proliferation was significantly decreased in 5AZA-treated uninfected and HCMV-infected cells compared with untreated uninfected and infected cells, respectively. ${ }^{*} \mathrm{P}<0.05,{ }^{* * *} \mathrm{P}<0.001,{ }^{* * * * *} \mathrm{P}<0.0001$. HCMV, human cytomegalovirus; GBM, glioblastoma; 5AZA, 5-azacytidine.

late proteins was low and rarely detected in GBM tissues, the expression of HCMV-IE was frequently detected at different levels in tumor cells and in the cells of blood vessel walls within the tumors. Furthermore, while DNMT-1 was expressed in the nuclei of tissue tumor cells, it was also present in the extranuclear space of cells in the vessel wall within the tumors (Fig. 4A and B depict two patients with primary GBM). In one GBM patient (patient number 2) who had deletion in chromosome 1p19q, DNMT-1 was expressed in the extranuclear space in the majority of tumor cells as well as in the vessel walls.

\section{Discussion}

HCMV latency has been unambiguously linked to epigenetic states by viral histone deacetylation, and viral reactivation to histone acetylation $(9,11,12,15)$. We previously reported that HCMV replication increased in DNA methylation-inhibited non-tumor (human umbilical vein endothelial cells) and tumor cells (MB) (25). However, in the present study, 5AZA treatment of HCMV-infected GBM cells did not significantly affect viral replication, although the treatment appeared to increase the number of cells expressing HCMV-IE proteins by $10 \%$.
This effect is likely due to the decreased number of cells as a result of 5AZA treatment, as untreated and 5AZA-treated cells were infected with an equal MOI of virus. As DNMT-1 and viral DNA transcript levels were not significantly altered by HCMV infection in U343 cells, the western blot analysis was not included. The opposite results on HCMV replication upon 5AZA treatment in MB (25) and GBM tumors suggests that the different biology and epigenetic mechanisms involved may affect the oncogenic signaling pathways. This distinction may be highly important when considering epigenetic therapies of cancers that harbor HCMV, such as MB and GBM. Furthermore, as we have previously reported for non-cancer cells and MB cells (25), infection of GBM cells by HCMV causes DNMT-1 accumulation in the cytoplasmic/extranuclear space, rather than the nucleus. This was found to be associated with the presence of HCMV late protein(s), as demonstrated by IHC in the present study.

As DNMT-1 is likely to only function in the nucleus (28), the DNMT inhibitor 5AZA was used to investigate the effect of reduced DNMT-1 activity on the cell proliferation and invasion. Both uninfected and HCMV-infected cells had evidently reduced proliferation following 5AZA treatment. HCMV infection of U343MG cells alone did not alter cell 
A
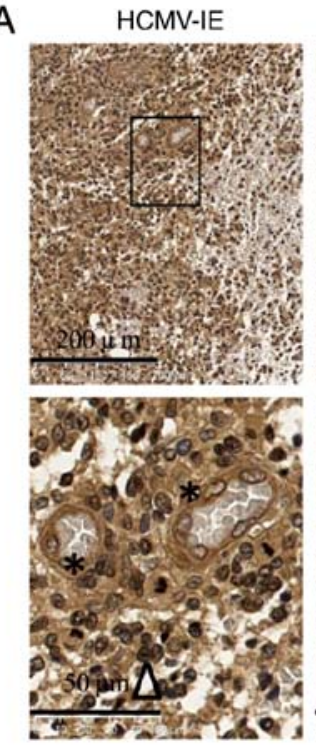

B
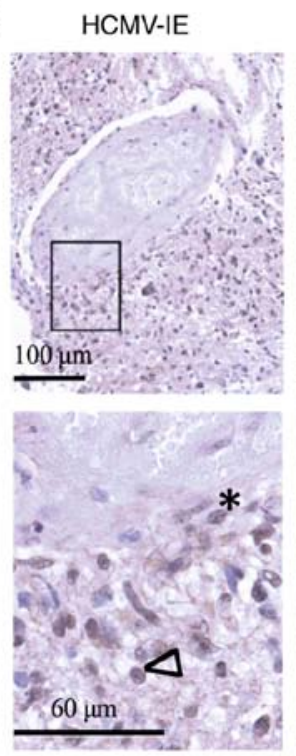

HCMV-Late
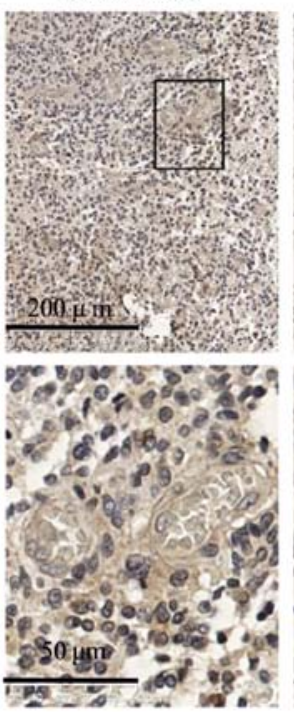

HCMV-Late
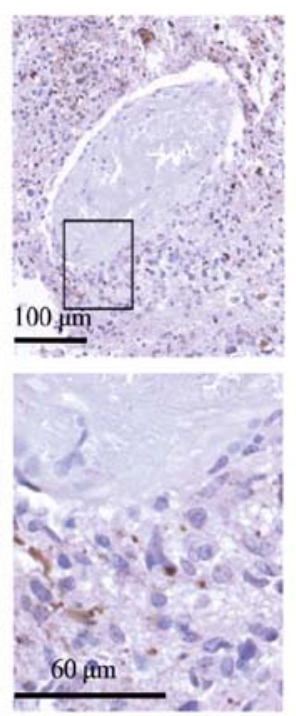

HCMV-gB
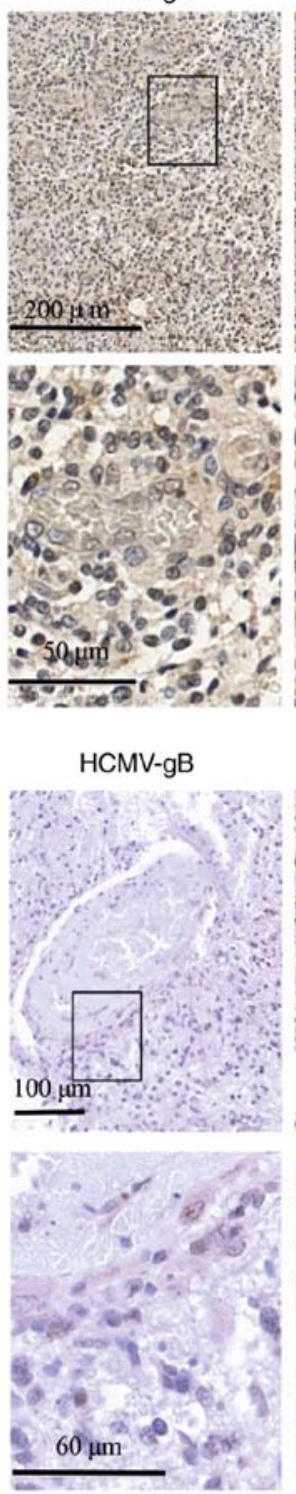
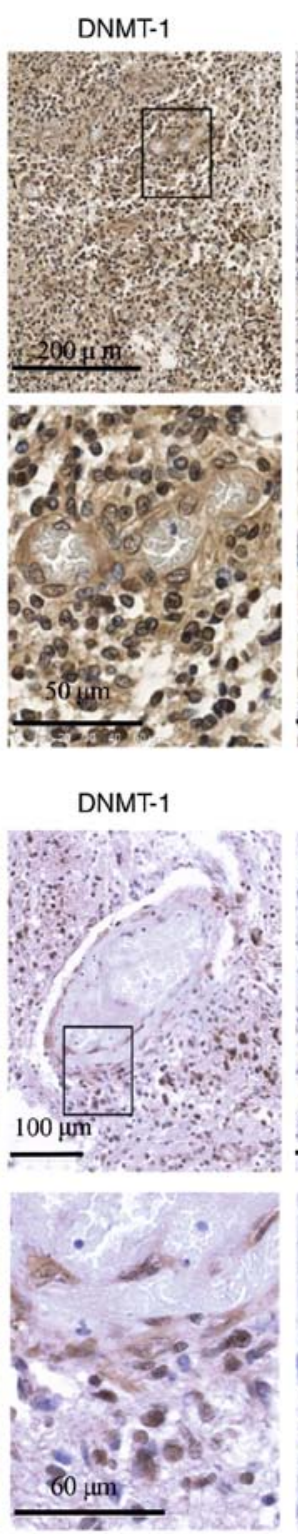

Negative control
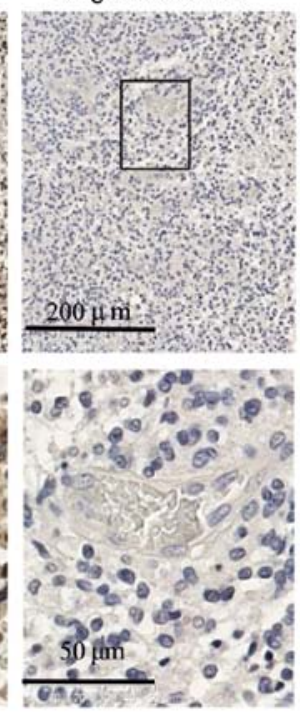

Negative control
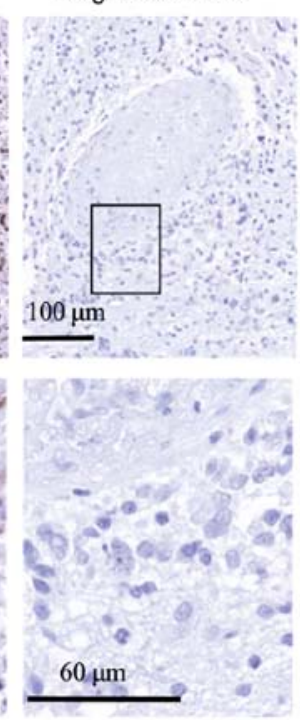

Figure 4. Detection of HCMV proteins in GBM tumor samples. (A and B) Expression of HCMV-IE, -late, -gB and DNMT-1 in cancer cells ( $\Delta$ ) and the cells of vessel walls (*) within the GBM tumor obtained from (A) primary GBM with chromosome 1p19q and (B) primary GBM patients. DNA methyltransferase 1; HCMV, human cytomegalovirus; GBM, glioblastoma; gB, glycoprotein gB; IE, immediate early.

proliferation, which has been previously reported in GBM cell lines (29). In contrast, the infection alone significantly decreased cell invasion, which was further reduced by 5AZA treatment. However, we have previously reported an increase in invasion by HCMV infection in U373MG cells (29). The differences in the invasion capacity of HCMV-infected GBM cells may be explained by different genetic background, global gene methylation status and infection rate, as cells were infected for 5 days in our previous study, compared to 3 days in the present study.

Whether or not the observed effects are related to DNMT relocalization or to other mechanisms requires further investigation. This information may be of importance when considering treatment of GBM patients with methylation inhibitors. However, since a majority of GBM tumors are likely to be infected by HCMV, a limitation of the present study is that there was no access HCMV negative GBM tissue sections. In our laboratory, the expression of various HCMV proteins in hundreds of GBM tissues have been detected, and only a limited number are HCMV protein negative. Therefore, HCMV-protein negative tissue specimens could not be examined for the current study.

We have previously reported extended overall survival in GBM patients receiving continued treatment with the antiviral drug Valcyte ${ }^{\circledR}$ as an add-on for routine treatment $(30,31)$. Hypothetically, based on the findings of the present study, GBM patients may benefit from a combination therapy including epigenetic drugs and Valcyte ${ }^{\circledR}$ with the aim of reducing the proliferation and invasion of tumor cells. This must, however, be thoroughly investigated prior to clinical trial investigation. Notably, cytoplasmic localization of DNMT-1 was predominantly observed in vessel walls, with or without HCMV-gB or pan late protein expression within the GBM tumor tissues. Further, in one GBM patient who had 
a co-deletion in chromosome $1 \mathrm{p} / 19 \mathrm{q}$, also known as oligodendroglioma, DNMT-1 was detected in the extranuclear space in the majority of tumor cells as well as in the vessel walls within the tumors. The contribution of the $1 \mathrm{p} / 19 \mathrm{q}$ deletion in oligodendroglioma oncogenesis is still unknown. GBM patients with $1 p / 19 q$ deletions are known to be chemosensitive with longer overall survival (OS) and progression free survival (PFS) regardless of the treatment they receive (32-36). Furthermore, the link between the $1 \mathrm{p} / 19 \mathrm{q}$ co-deletion and epigenetic alterations in the course of demethylation/hypomethylation has previously been studied. A positive association between isocitrate dehydrogenase (IDH) promoter mutations and oncometabolite R-2-hydroxyglutarate (R-2-HG) production was reported $(37,38)$. Various enzymes, including epigenetic regulatory methylcytosine dioxygenase TET (TET)2, are inhibited by R-2-HG, causing hypermethylation of DNA $(37,38)$. However, in the present study, DNMT-1 was localized in the cytoplasm of many tumor cells and the cells of vessel walls within the tumor in the patient with $1 \mathrm{p} / 19 \mathrm{q}$ co-deletion. Additionally, in this patient, the methylated-DNA-protein-cysteine methyltransferase (MGMT) promoter was unmethylated and tumor regrowth occurred at 8 months, resulting in an overall survival of only 14.5 months. Of note, HCMV-IE protein was frequently expressed at various levels both in the tumor cells and in the cells of vessel walls within the GBM tumors examined in this study. In an in vivo scenario in GBM tumors where HCMV-IE is frequently expressed while HCMV-late genes are rarely expressed, a direct or indirect effect of HCMV-IE expression on DNMT-1 localization, increased tumor cell proliferation, and invasion cannot be excluded. Hypothetically, the absence of nuclear and presence of extranuclear localization of DNMTs in HCMV-infected vessel cells in the GBM tissues may lead to demethylation/hypomethylation of DNA and subsequent alteration of cellular characteristics, as well as potential induction of factors that impact angiogenesis and tumorigenesis. Furthermore, exposure of the cells to inflammatory factors and cytokines such as prostaglandin E2 (PGE2), interleukin (IL)-1, IL-6 and IL-8, alters levels and/or activity of enzymes involved in DNA and histone methylation, resulting in global and potentially gene-specific changes which could in turn lead to changes in cellular phenotypes over time, resulting in increased invasion and proliferation $(39,40)$. Furthermore, increased production of inflammatory factors and cytokines such as PGE2, IL-2, IL-6 and IL- 8 in response to HCMV infection have previously been reported $(41,42)$.

In the present study, the proliferation and invasion ability of uninfected and HCMV infected U343MG cells with or without 5AZA treatment was investigated. A significant decrease in the proliferation of both treated uninfected as well as infected cells was detected. Conversely, invasion capacity was significantly decreased both by infection alone, as well as by 5AZA treatment in uninfected and infected cells. Based on this observation, the potential benefits of this treatment in HCMV-infected GBM patients to inhibit tumor invasion and proliferation should be further investigated.

The importance of DNA methylation in GBM is suggested by previous studies, which have reported hypomethylation of oncogenes and hypermethylation of tumor suppressor genes in GBM (43-46). Nagarajan and Costello (44) reported hypomethylation in 76 promoters including TERT and tumor protein 73 , with subsequent increased transcript expression, suggesting that alterations in the gene regulatory mechanisms in GBM lead to the expression of oncogenic proteins. Hypermethylated suppressor genes in GBM include RB transcriptional corepressor 1, epithelial membrane protein 3, Ras association domain family member 1 isoform A (RASSF1A), cadherin 1 (CDH1) and zinc finger MYND-type containing 10, cell cycle regulators [cyclin dependent kinase inhibitor 2 (CDKN2)A and CDKN2B], DNA repair genes (MGMT, MutL homolog 1), and genes involved in tumor invasion and apoptosis [death-associated protein kinase 1 (DAPK) and TIMP metallopeptidase inhibitor 3] (44,46-54). However, gene specific methylation analysis should be performed in order to clarify the effects of HCMV infection on hyper- or hypomethylation of these genes. We performed gene specific methylation analyses of CDKNA, RASSF1A, MGMT, DAPK and CDH1 with a multiplex methylation specific PCR (MMSP) (55) assay in untreated, 5AZA treated, uninfected, and HCMV infected U343 MG cells. It was shown that all examined genes under the different conditions were hypermethylated, excluding CDKNA and DAPK, which were hypomethylated under all different conditions (data not shown). Optimization of a more accurate quantification method of gene hypermethylation is required. It is likely that there are heterogeneities in the methylation for several of these genes, but the method used is not linear with the amount of methylation, and therefore very small amounts of specific methylation may be revealed as greater than they are. In the future, these experiments will be performed in further detail.

As previously mentioned, extranuclear localization of DNMT-1 was observed in the cells of vessel walls within the GBM tumor tissues, as well as in the tumor cells of GBM tissues examined from a patient with co-deletion on chromosome 1p/19q. This may lead to altered cellular functions by demethylation of certain genes, resulting in a more oncogenic phenotype of these cells. It is of note that temozolomide treatment (alkylating agent) is more effective in GBM patients with a hypermethylated (downregulated) MGMT gene, which codes for a DNA repair enzyme (56,57). In GBM patients, MGMT promoter methylation is used as a biomarker for clinical temozolomide therapy management. Furthermore, hypermethylation and mutations in IDH1/2 with functional consequences for TET enzymes (involved in the demethylation of histones and DNA) as well as glial differentiation and establishment of glioma, have been reported (58). Since hypermethylation and silencing of tumor suppressors are frequently observed in cancer $(59,60)$, and due to the reversible nature of epigenetic mechanisms, these are potential therapeutic cancer targets (61).

In conclusion, it was demonstrated that HCMV-infected GBM cells relocalize their DNMT-1 from the nucleus to the extranuclear space, which coincided with viral late protein production. The treatment of GBM cells with 5AZA resulted in reduced cell proliferation. In addition, cell invasion was decreased by infection. In vivo, most GBM tumors exhibited cytoplasmic localization of DNMT-1, predominantly in blood vessel cells, whereas cancer cells retained DNMT-1 expression in the nuclei. These findings may be of importance in further investigations into using DNA methylation and viral inhibitors in GBM therapy. 


\section{Acknowledgements}

Not applicable.

\section{Funding}

The Swedish Cancer Foundation, The Children's Cancer Foundation of Sweden, Swedish Society for Medical Research (SLS), Goljes Memory Foundation, Magnus Bergvall Foundation, Swedish Society for Medical Research (SSMF), Karolinska Institute and Tore Nilsson Foundation for Medical Research.

\section{Availability of data and materials}

The datasets used and/or analyzed during the present study are available from the corresponding author on reasonable request.

\section{Authors' contributions}

AR and TJE designed the study; AE, NL, BD and AR performed the experiments; GS provide clinical samples and data; AR and AE analyzed and generated figures; AR and TJE wrote the manuscript. MRP, IN and LFH performed methylation experiments. All authors read and approved the manuscript and agree to be accountable for all aspects of the research in ensuring that the accuracy or integrity of any part of the work are appropriately investigated and resolved.

\section{Ethical approval and consent to participate}

Ethical permission was approved by local ethical committee at Karolinska Institute, Sweden (Dnr. 2008/628-31).

\section{Patient consent for publication}

Not applicable.

\section{Competing interests}

The authors declare that they have no competing interests.

\section{References}

1. Dziurzynski K, Chang SM, Heimberger AB, Kalejta RF, McGregor Dallas SR, Smit M, Soroceanu L and Cobbs CS; HCMV and Gliomas Symposium: Consensus on the role of human cytomegalovirus in glioblastoma. Neuro Oncol 14: 246-255, 2012.

2. Cunningham C, Gatherer D, Hilfrich B, Baluchova K, Dargan DJ, Thomson M, Griffiths PD, Wilkinson GW, Schulz TF and Davison AJ: Sequences of complete human cytomegalovirus genomes from infected cell cultures and clinical specimens. J Gen Virol 91: 605-615, 2010.

3. Dunn W, Chou C, Li H, Hai R, Patterson D, Stolc V, Zhu H and Liu F: Functional profiling of a human cytomegalovirus genome. Proc Natl Acad Sci USA 100: 14223-14228, 2003.

4. Slobedman B and Mocarski ES: Quantitative analysis of latent human cytomegalovirus. J Virol 73: 4806-4812, 1999.

5. Bego MG, Keyes LR, Maciejewski J and St Jeor SC: Human cytomegalovirus latency-associated protein LUNA is expressed during HCMV infections in vivo. Arch Virol 156: 1847-1851, 2011.
6. Wills MR, Poole E, Lau B, Krishna B and Sinclair JH: The immunology of human cytomegalovirus latency: Could latent infection be cleared by novel immunotherapeutic strategies? Cell Mol Immunol 12: 128-138, 2015.

7. Goodrum F: Human cytomegalovirus latency: Approaching the gordian knot. Annu Rev Virol 3: 333-357, 2016.

8. Soderberg-Naucler C, Fish KN and Nelson JA: Reactivation of latent human cytomegalovirus by allogeneic stimulation of blood cells from healthy donors. Cell 91: 119-126, 1997.

9. Liu XF, Wang X, Yan S, Zhang Z, Abecassis M and Hummel M: Epigenetic control of cytomegalovirus latency and reactivation. Viruses 5: 1325-1345, 2013.

10. Sinclair J: Human cytomegalovirus: Latency and reactivation in the myeloid lineage. J Clin Virol 41: 180-185, 2008.

11. Gan X, Wang H, Yu Y, Yi W, Zhu S, Li E and Liang Y: Epigenetically repressing human cytomegalovirus lytic infection and reactivation from latency in THP-1 model by targeting H3K9 and H3K27 histone demethylases. PLoS One 12: e0175390, 2017.

12. Sinclair J: Chromatin structure regulates human cy tomegalovirus gene expression during latency, reactivation and lytic infection. Biochim Biophys Acta 1799: 286-295, 2010.

13. Meier JL: Reactivation of the human cytomegalovirus major immediate-early regulatory region and viral replication in embryonal NTera2 cells: Role of trichostatin A, retinoic acid, and deletion of the 21-base-pair repeats and modulator. J Virol 75: 1581-1593, 2001.

14. Ioudinkova E, Arcangeletti MC, Rynditch A, De Conto F, Motta F, Covan S, Pinardi F, Razin SV and Chezzi C: Control of human cytomegalovirus gene expression by differential histone modifications during lytic and latent infection of a monocytic cell line. Gene 384: 120-128, 2006.

15. Murphy JC, Fischle W, Verdin E and Sinclair JH: Control of cytomegalovirus lytic gene expression by histone acetylation. EMBO J 21: 1112-1120, 2002.

16. Baryawno N, Rahbar A, Wolmer-Solberg N, Taher C, Odeberg J, Darabi A, Khan Z, Sveinbjörnsson B, FuskevÅg OM, Segerström L, et al: Detection of human cytomegalovirus in medulloblastomas reveals a potential therapeutic target. J Clin Invest 121: 4043-4055, 2011.

17. Rahbar A, Orrego A, Peredo I, Dzabic M, Wolmer-Solberg N, Strååt K, Stragliotto G and Söderberg-Nauclér C: Human cytomegalovirus infection levels in glioblastoma multiforme are of prognostic value for survival. J Clin Virol 57: 36-42, 2013.

18. Wolmer-Solberg N, Baryawno N, Rahbar A, Fuchs D, Odeberg J, Taher C, Wilhelmi V, Milosevic J, Mohammad AA, Martinsson T, et al: Frequent detection of human cytomegalovirus in neuroblastoma: A novel therapeutic target? Int J Cancer 133: 2351-2361, 2013.

19. Stupp R, Mason WP, van den Bent MJ, Weller M, Fisher B, Taphoorn MJ, Belanger K, Brandes AA, Marosi C, Bogdahn U, et al: Radiotherapy plus concomitant and adjuvant temozolomide for glioblastoma. N Engl J Med 352: 987-996, 2005.

20. Johnson DR, Fogh SE, Giannini C, Kaufmann TJ, Raghunathan A, Theodosopoulos PV and Clarke JL: Case-based review: Newly diagnosed glioblastoma. Neuro Oncol Pract 2: 106-121, 2015.

21. Alifieris $\mathrm{C}$ and Trafalis DT: Glioblastoma multiforme: Pathogenesis and treatment. Pharmacol Ther 152: 63-82, 2015.

22. Tyagi V, Theobald J, Barger J, Bustoros M, Bayin NS, Modrek AS, Kader M,AndererEG,Donahue B,Fatterpekar G, et al: Traumatic brain injury and subsequent glioblastoma development: Review of the literature and case reports. Surg Neurol Int 7: 78, 2016.

23. Herbein G: The human cytomegalovirus, from oncomodulation to oncogenesis. Viruses 10: pii: E408, 2018.

24. Michaelis M, Baumgarten P, Mittelbronn M, Driever PH, Doerr HW and Cinatl J Jr: Oncomodulation by human cytomegalovirus: Novel clinical findings open new roads. Med Microbiol Immunol 200: 1-5, 2011.

25. Estekizadeh A, Landazur N, Bartek J Jr, Beltoft Brøchner C, Davoudi B, Broholm H, Karimi M, Ekström TJ and Rahbar A: Increased cytomegalovirus replication by 5 -Azacytidine and viral-induced cytoplasmic expression of DNMT1 in medulloblastoma and endothelial cells. Int J Oncol 52: 1317-1327, 2018.

26. Di Costanzo A, Del Gaudio N, Migliaccio A and Altucci L: Epigenetic drugs against cancer: An evolving landscape. Arch Toxicol 88: 1651-1668, 2014.

27. Livak KJ and Schmittgen TD: Analysis of relative gene expression data using real-time quantitative PCR and the $2^{-\Delta \Delta C_{\mathrm{T}}}$ method. Methods 25: 402-408, 2001. 
28. (Human protein Atlas: https://www.proteinatlas.org/ ENSG00000130816-DNMT1/cell). Version: 18.1, Atlas updated: 2018-11-15.

29. Khan Z, Yaiw KC, Wilhelmi V, Lam H, Rahbar A, Stragliotto G and Söderberg-Nauclér C: Human cytomegalovirus immediate early proteins promote degradation of connexin 43 and disrupt gap junction communication: Implications for a role in gliomagenesis. Carcinogenesis 35: 145-154, 2014.

30. Stragliotto G, Rahbar A, Solberg NW, Lilja A, Taher C, Orrego A Bjurman B, Tammik C, Skarman P, Peredo I, et al: Effects of valganciclovir as an add-on therapy in patients with cytomegalovirus-positive glioblastoma: A randomized, double-blind, hypothesis-generating study. Int J Cancer 133: 1204-1213, 2013.

31. Soderberg-Naucler C, Rahbar A and Stragliotto G: Survival in patients with glioblastoma receiving valganciclovir. $\mathrm{N}$ Engl J Med 369: 985-986, 2013.

32. Bettegowda C, Agrawal N, Jiao Y, Sausen M, Wood LD, Hruban RH, Rodriguez FJ, Cahill DP, McLendon R, Riggins G, et al: Mutations in CIC and FUBPI contribute to human oligodendroglioma. Science 333: 1453-1455, 2011.

33. Bibikova M, Barnes B, Tsan C, Ho V, Klotzle B, Le JM, Delano D Zhang L, Schroth GP, Gunderson KL, et al: High density DNA methylation array with single $\mathrm{CpG}$ site resolution. Genomics 98 : 288-295, 2011.

34. Brennan CW, Verhaak RG, McKenna A, Campos B Noushmehr H, Salama SR, Zheng S, Chakravarty D, Sanborn JZ, Berman SH, et al: The somatic genomic landscape of glioblastoma. Cell 155: 462-477, 2013.

35. Ahluwalia MS, Xie H, Dahiya S, Hashemi-Sadraei N, Schiff D, Fisher PG, Chamberlain MC, Pannullo S, Newton HB, Brewer C, et al: Efficacy and patient-reported outcomes with dose-intense temozolomide in patients with newly diagnosed pure and mixed anaplastic oligodendroglioma: A phase II multicenter study. J Neurooncol 122: 111-119, 2015.

36. Vogelbaum MA, Hu C, Peereboom DM, Macdonald DR Giannini C, Suh JH, Jenkins RB, Laack NN, Brachman DG, Shrieve DC, et al: Phase II trial of pre-irradiation and concurrent temozolomide in patients with newly diagnosed anaplastic oligodendrogliomas and mixed anaplastic oligoastrocytomas: Long term results of RTOG BR0131. J Neurooncol 124: 413-420, 2015.

37. Aihara K, Mukasa A, Nagae G, Nomura M, Yamamoto S, Ueda H, Tatsuno K, Shibahara J, Takahashi M, Momose T, et al: Genetic and epigenetic stability of oligodendrogliomas at recurrence. Acta Neuropathol Commun 5: 18, 2017.

38. Suvà ML: Genetics and epigenetics of gliomas. Swiss Med Wkly 44: w14018, 2014

39. Li X, Zhang Q, Shi Q, Liu Y, Zhao K, Shen Q, Shi Y, Liu X, Wang C, Li N, et al: Demethylase Kdm6a epigenetically promotes IL- 6 and IFN- $\beta$ production in macrophages. J Autoimmun 80 : 85-94, 2017.

40. Winfield J, Esbitt A, Seutter SF, Desai B, Abdo M, Vasconez M, Laidlaw W, Green K, Shamseddin SM and Borghaei RC: Effect of inflammatory cytokines on DNA methylation and demethylation. FASEB J 30 (Suppl 1): S1053, 2016.

41. Cheeran MC, Hu S, Yager SL, Gekker G, Peterson PK and Lokensgard JR: Cytomegalovirus induces cytokine and chemokine production differentially in microglia and astrocytes: Antiviral implications. J Neurovirol 7: 135-147, 2001.

42. Kline JN, Hunninghake GM, He B, Monick MM and Hunninghake GW: Synergistic activation of the human cytomegalovirus major immediate early promoter by prostaglandin E2 and cytokines. Exp Lung Res 24: 3-14, 1998.

43. Gong M, Shi W, Qi J, Shao G, Shi Z, Wang J, Chen J and Chu R: Alu hypomethylation and MGMT hypermethylation in serum as biomarkers of glioma. Oncotarget 8: 76797-76806, 2017.

44. Nagarajan RP and Costello JF: Epigenetic mechanisms in glioblastoma multiforme. Semin Cancer Biol 19: 188-197, 2009.
45. Cadieux B, Ching TT, VandenBerg SR and Costello JF Genome-wide hypomethylation in human glioblastomas associated with specific copy number alteration, methylenetetrahydrofolate reductase allele status, and increased proliferation. Cancer Res 66: 8469-8476, 2006.

46. Costello JF, Berger MS, Huang HS and Cavenee WK: Silencing of p16/CDKN2 expression in human gliomas by methylation and chromatin condensation. Cancer Res 56: 2405-2410, 1996.

47. Alaminos M, Davalos V, Ropero S, Setién F, Paz MF, Herranz M, Fraga MF, Mora J, Cheung NK, Gerald WL, et al: EMP3, a myelin-related gene located in the critical 19q13.3 region, is epigenetically silenced and exhibits features of a candidate tumor suppressor in glioma and neuroblastoma. Cancer Res 65: 2565-2571, 2005.

48. Nakamura M, Watanabe T, Yonekawa Y, Kleihues $\mathrm{P}$ and Ohgaki H: Promoter methylation of the DNA repair gene MGMT in astrocytomas is frequently associated with G:C $\rightarrow$ A:T mutations of the TP53 tumor suppressor gene. Carcinogenesis 22: $1715-1719,2001$

49. Hesson L, Bieche I, Krex D, Criniere E, Hoang-Xuan K, Maher ER and Latif F: Frequent epigenetic inactivation of RASSF1A and $B L U$ genes located within the critical 3p21.3 region in gliomas. Oncogene 23: 2408-2419, 2004.

50. Uhlmann K, Rohde K, Zeller C, Szymas J, Vogel S, Marczinek K, Thiel G, Nürnberg P and Laird PW: Distinct methylation profiles of glioma subtypes. Int J Cancer 106: 52-59, 2003.

51. Nakamura M, Yonekawa Y, Kleihues P and Ohgaki H: Promoter hypermethylation of the RB1 gene in glioblastomas. Lab Invest 81: 77-82, 2001

52. Hegi ME, Diserens AC, Gorlia T, Hamou MF, de Tribolet N, Weller M, Kros JM, Hainfellner JA, Mason W, Mariani L, et al: $M G M T$ gene silencing and benefit from temozolomide in glioblastoma. N Engl J Med 352: 997-1003, 2005.

53. Gonzalez-Gomez P, Bello MJ, Arjona D, Lomas J, Alonso ME, De Campos JM, Vaquero J, Isla A, Gutierrez M and Rey JA: Promoter hypermethylation of multiple genes in astrocytic gliomas. Int J Oncol 22: 601-608, 2003.

54. Alonso ME, Bello MJ, Gonzalez-Gomez P, Arjona D, Lomas J, de Campos JM, Isla A, Sarasa JL and Rey JA: Aberrant promoter methylation of multiple genes in oligodendrogliomas and ependymomas. Cancer Genet Cytogenet 144: 134-142, 2003.

55. Nawaz I, Qiu X, Wu H, Li Y, Fan Y, Hu LF, Zhou Q and Ernberg I: Development of a multiplex methylation specific PCR suitable for (early) detection of non-small cell lung cancer. Epigenetics 9: $1138-1148,2014$

56. Paz MF, Yaya-Tur R, Rojas-Marcos I, Reynes G, Pollan M, Aguirre-Cruz L, García-Lopez JL, Piquer J, Safont MJ, Balaña $\mathrm{C}$, et al: $\mathrm{CpG}$ island hypermethylation of the DNA repair enzyme methyltransferase predicts response to temozolomide in primary gliomas. Clin Cancer Res 10: 4933-4938, 2004.

57. Caren H, Pollard SM and Beck S: The good, the bad and the ugly: Epigenetic mechanisms in glioblastoma. Mol Aspects Med 34: 849-862, 2013

58. Flanagan S, Lee M, Li CC, Suter CM and Buckland ME: Promoter methylation analysis of IDH genes in human gliomas. Front Oncol 2: 193, 2012.

59. Hughes LA, Melotte V, de Schrijver J, de Maat M, Smit VT, Bovée JV, French PJ, van den Brandt PA, Schouten LJ, de Meyer T, et al: The CpG island methylator phenotype: What's in a name? Cancer Res 73: 5858-5868, 2013.

60. Dong Y, Zhao H, Li H, Li X and Yang S: DNA methylation as an early diagnostic marker of cancer (Review). Biomed Rep 2: 326-330, 2014.

61. Fardi M, Solali S and Farshdousti Hagh M: Epigenetic mechanisms as a new approach in cancer treatment: An updated review. Genes Dis 5: 304-311, 2018. 